Proceedings

\title{
A mixed-source approach to public participation for assessing urban accessibility of neighbourhood public spaces
}

1 Building Sciences and Urbanism Department, University of Alicante; mh65@alu.ua.es

2 Building Sciences and Urbanism Department, University of Alicante; alvaro.bautista@ua.es

3 Building Sciences and Urbanism Department, University of Alicante; leticia.serrano@ua.es

* Correspondence: mh65@alu.ua.es

Citation: Huskinson, M.; Bernabeu-Bautista, A..; Serrano-Estrada, L., 2022, A mixed-source approach to public participation for assessing urban accessibility of neighbourhood public spaces. SUPTM 2022 conference proceedings sciforum-054432. https://doi.org/10.31428/10317/10494

Publisher's Note: UPCT and Sciforum stays neutral with regard to jurisdictional claims in published maps and institutional affiliations.

Copyright: (c) 2022 by the authors. Submitted for possible open access publication under the terms and conditions of the Creative Commons Attribution (CC BY) license (https://creativecommons.org/license s/by/4.0/).

\begin{abstract}
An accessible city is one that provides high-quality public spaces to facilitate gatherings, access to services, and comfortable journeys for people of all ages and abilities. In order to achieve such spaces, public participation is crucial for planning, urban policy formulation and decision making that ultimately have a direct impact on the city. This research aims to build on the understanding of individual and collective needs in terms of the perception of accessibility to urban public spaces. A mixed-source approach to public participation is proposed that combines data gathered from direct citizen participation and passively crowdsourced geolocated data. Two neighbourhoods in Alicante (Spain) are adopted as a case study. Findings demonstrate the potential of a mixed-source public participation approach to delve deeper into the public spaces' current status by identifying environmental, physical and perceptual issues that impact on the quality of the cities' everyday experience.
\end{abstract}

Keywords: Urban accessibility, public spaces, neighbourhood, crowdsourced data, field studies.

\section{Introduction}

Urban accessibility is currently a highly relevant pillar for achieving the Sustainable Development Goals of the 2030 Agenda. Specifically, this research addresses the following goals: 5- Gender Equality, 10- Reduction of inequalities and 11- Sustainable cities and communities. In this sense, the general interest from various cities to improve urban accessibility as a priority action is noteworthy. Precisely, accessibility has been studied for many different objectives [1] from the improvement of transport infrastructures to the elimination of physical barriers that prevent equal and universal access to public spaces. To this end, various guides of good practices have been developed over the years for the creation of urban environments suitable for all ages and abilities [2-4]. For example, environmental assessment tools focusing on older people [5] or people with disabilities [6,7] among others. They make recommendations regarding the built environment based primarily on empirical data obtained through field studies [8].

This research aims to contribute to this line of work focusing on the perception of accessibility to urban public spaces. This study is oriented more towards the capacity of the built environment to be perceived and valued as accessible by users than towards the analysis of its physical characteristics. To this end, in addition to the empirical work of field data collection through interviews, it is proposed to introduce complementary methodologies to those of a more traditional nature in citizen participation: geolocated social media. These sources have shown their potential to enrich, as well as facilitate the collection of information related to perception [8], citizen preferences [9], usage patterns [10], urban activities [11] and presence in urban spaces [12].

\subsection{Objectives}

This research is based on the notion that citizen participation is an extremely relevant pillar to identify and diagnose key aspects in the collective perception of the accessibility 
of public spaces. In this sense, the aim of this research is to examine and compare two different participation methodologies as tools for assessing the perceptual accessibility of public spaces.

\section{Methodology}

A methodological approach is proposed by introducing information from complementary sources of information based on direct approaches - surveys of groups, fieldwork and interviews - and less direct approaches, such as user-generated data from social media and web services. Therefore, the method designed for this research is divided into two parts (Figure 1).

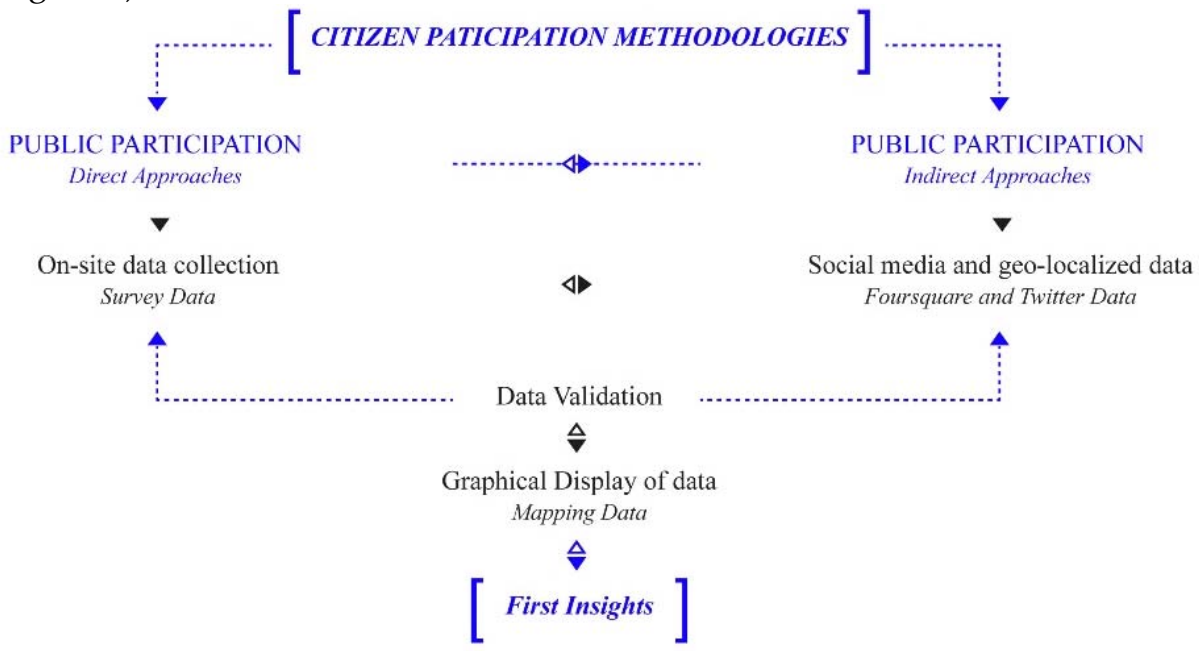

Figure 1. Methodological flowchart.

The city of Alicante was selected as case study. It is located in the province of Alicante, with the highest rate of population with disabilities in the Valencian Community (INE, 2008).

The method consisted mainly in two phases. In the first phase (direct approach) the objective was to identify two case study neighbourhoods with public spaces that are perceived as less accessible. A survey was created in Google Forms and publicly shared in several social networks. They were also conducted through interviewers placed in public spaces. In these surveys citizens provided information related to the city's public space conditions in terms of accessibility. Also, they would have to indicate in the survey which neighbourhoods of the city were accessible or not according to their experience. The selected neighbourhoods are Carolinas and Campoamor, the ones from which the most negative opinions were obtained in terms of accessibility from the surveys.

For the second phase (indirect approach) two social networks were selected: Foursquare and Twitter. The data from these two sources are considered as indicators of urban activity and preferences, and citizen opinions and presence, respectively. The dates from the data retrieved from these sources are: Foursquare - 20th October 2021 and Twitter 16 March 2020 to 30 September 2020.

\section{Results}

\subsection{Results from the direct approach public participation}

\subsubsection{Surveys}

In total, 200 people from the city of Alicante answered the survey, among which $12.6 \%$ are disabled population and $15.9 \%$ are people over 55 years old. From the surveys diverse information was obtained, from which it is worth highlighting the evaluation of the degree of accessibility of the neighbourhoods of the city of Alicante, as well as the 
location of the public spaces mentioned and perceived as more or less accessible by surveyed citizens. The results obtained indicate that the Carolinas neighbourhood is $66.70 \%$ inaccessible and Campoamor 100\% inaccessible. From these neighbourhoods, it was possible to distinguish between those public spaces that are perceived as accessible or inaccessible according to the public opinion. For instance, Plaza Pío XII (Carolinas) stands out for obtaining the best positive evaluation (Accessible) and Plaza Castellón (Carolinas) for having the worst evaluation (Inaccessible).

\subsection{Public participation methodologies: Indirect approach}

\subsubsection{Foursquare}

Foursquare data provides information about the establishments in the city that have been registered at least once, therefore the establishments registered on the social network are considered relevant to society [13]. These establishments are classified into 10 different categories. For this research, establishments under the category "Outdoors and Recreation" were selected as it includes a list of public spaces in a specific area. With such data it was possible to quantify the number of users that have registered their presence in the social network while being physically in each of the urban public spaces, obtaining similar values as those from people-counting methods. The venues with the highest number of registered users are located near the Escaleritas Jose Rico Pérez, Plaza San Juan de Dios, Pasaje Duval and with the highest number in Plaza América (Campoamor neighbourhood). Regarding Carolinas neighbourhood, the venues with the highest number of registered users are located near Plaza Pío XII and to a lower degree Plaza del Sol (Figure 2).

\subsubsection{Twitter}

Twitter data enables a comparison of user activity that can help to monitor the presence of citizens in urban public spaces (Figure 2). Applied to our case studies, the data provided by citizens do not determine any specific pattern of presence. On the other hand, if this data is analysed on a larger scale, we can see a concentration of tweets shared in areas where the activities available are far wider, such as Alicante city centre, for example.

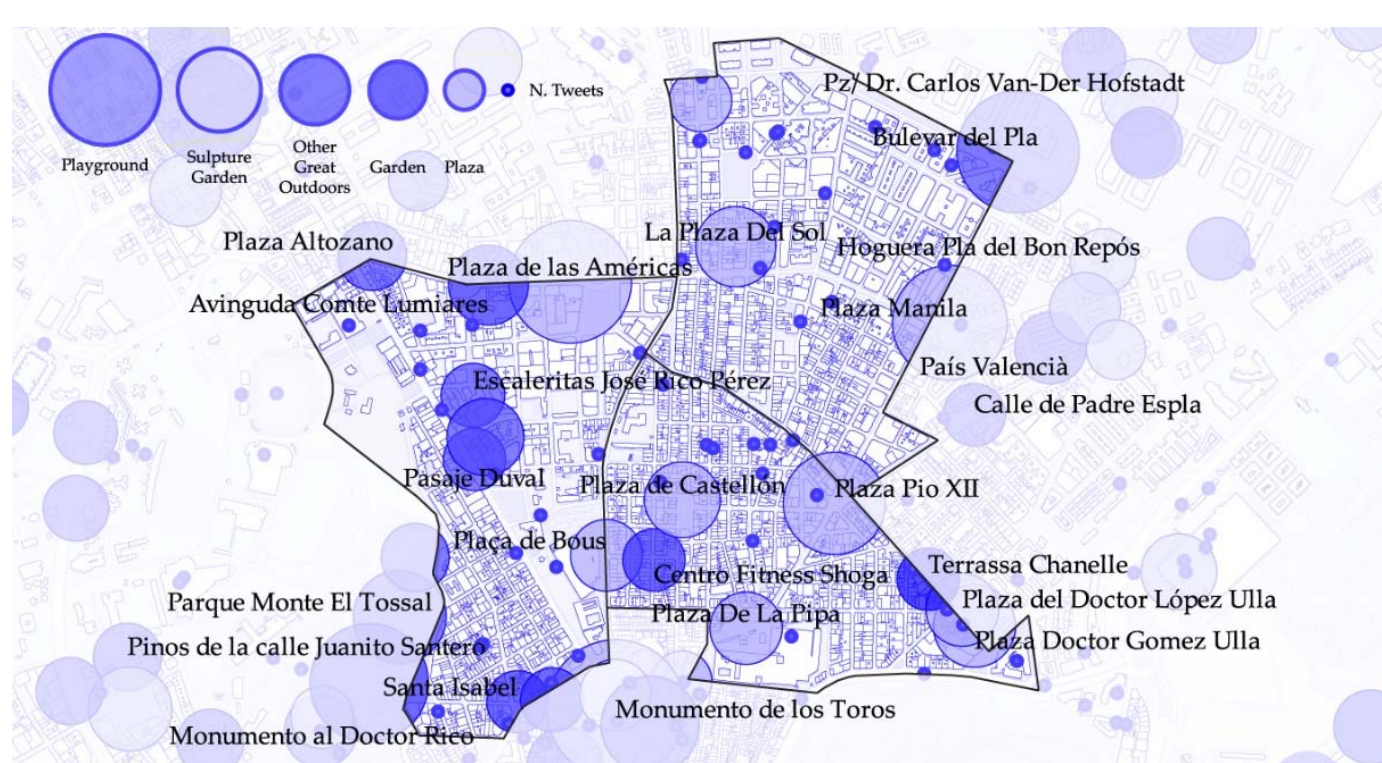

Figure 2. Visualization of Foursquare and Twitter data. The circles with different colours indicate different categories from "Outdoors and Recreation" data. The size of the circles indicates the number of users registered at those specific locations, the larger the size the more users. Smaller dots reflect the location of tweets shared.

\section{Discussion and Conclusions}

From the research conducted, it has been demonstrated that the polarisation of activities and the presence of citizens in urban public spaces according to social media data 
occurs on those spaces that are perceived as accessible according to the surveys. This research contributes to the existing knowledge on the complementary potential traditional fieldwork citizen participation-based sources and user-generated social network-based information for identifying social needs and reading people's perception about the accessibility of public spaces. The data obtained from the social networks show, in a representative way, what happens in the physical dimension. For the future line of research, a more exhaustive and specific study will be carried out in order to reach a fine-grain human scale detail to pinpoint those aspects that contribute to the "perceptual" urban accessibility.

Funding: This research was co-funded by (i) Conselleria de Innovación, Universidades, Ciencia y Sociedad Digital, Generalitat Valenciana (GV/2021/177) and (ii) Conselleria de Innovación, Universidades, Ciencia y Sociedad Digital, Generalitat Valenciana and European Social Fund (ACIF/2020/173).

Conflicts of Interest: The authors declare no conflict of interest.

\section{References}

